\title{
Code generation and recognition using a modified ejection system in die-casting process
}

\author{
Jaekyung Song ${ }^{1,2}$, Jeongsu Lee ${ }^{2}$, Young Chul Lee', Byoung Jun Lee', *Chaeho Lim ${ }^{1,2}$ \\ 1. Industrial Materials and Smart Manufacturing Engineering, Korea University of Science and Technology, Daejeon City, \\ Republic of Korea \\ 2. Digital Manufacturing Process Group, Korea Institute of Industrial Technology, Gyeonggi-do, Republic of Korea
}

\begin{abstract}
A code-generation and recognition technology that uses a modified ejection system in the diecasting process is presented. To achieve the highest level of quality management, the first requirement in the manufacturing process is to establish a product management system according to the specific product unit. Thus, a method to individually identify each product, such as a barcode or QR code, is required during the production process. Products manufactured in the die-casting process always have ejector pin (EP) marks. Herein, an ejection system was modified to generate a unique code using EP marks. This ejection system has two features: an EP with a modified head to show the direction of rotation, and a function to dependently rotate EPs (five or six EPs) with a constant angle. The EPs are numbered according to the rotation angle. Thus, the EP marks can be viewed as a five- or six-digit code. A program was also developed to individually identify the products by automatically detecting and reading the EPs using deep learning-based object detection and classification technology.
\end{abstract}

Key words: code recognition; die-casting; ejection system; object detection; product management; smart manufacturing
CLC numbers: TP391.9
Document code: A
Article ID: 1672-6421(2020)05-364-08

$\mathrm{T}$ The die-casting process is used to produce metal parts or products that are used in the manufacture of aircraft, automobiles, and ships, as well as for various types of mechanical equipment. Improvements in the die-casting process will lead to higher quality and stability of products manufactured throughout the industry. In recent years, considerable efforts have been made to equip industry with smart processes that utilize the Internet of Things, big data analysis, and artificial intelligence at various industrial sites.

The authors are conducting various pieces of research on smart die-casting processes. In particular, they are attempting to establish a smart system that can operate at low cost in small and medium-sized enterprises. A significant advantage of the smart process is the reduction in defect rate, which ensures

\footnotetext{
*Chaeho Lim

Male, born in 1970, Ph.D., Principle Researcher. Research interests: numerical simulation of the casting process, additive manufacturing of metals, industrial computed tomography technology, and smart manufacturing technology for small \& medium factories.

E-mail: chlim@kitech.re.kr
}

Received: 2020-03-09; Accepted: 2020-06-21 manufacture of stable and high-quality products while reducing costs. Currently, most factories only record the defect rate after the products have been inspected and directly removed by a human during the manufacturing process. Such statistical management is neither accurate nor precise in determining the cause of the defect. Smart processes, however, require interpretation and recording of an individual product unit, not only statistical quality management on a production basis. As the first step to achieving this, the product must be individually identified using product identification numbers (barcodes or QR codes) that are attached to the finished product. However, engraving identification numbers on metal products during the production process is difficult as the products are generated at extremely high temperatures (above $600{ }^{\circ} \mathrm{C}$ ); this would require various processes, e.g., surface treatment. Currently, the commonly used method is laser marking, which uses a laser to carve the identification numbers or QR codes onto the metal surface ${ }^{[1-3]}$. However, laser marking is not easy to use in small and medium-sized manufacturing enterprises owing to the additional cost of the expensive equipment and production line. Furthermore, the production line 
addition also causes a slight decrease in productivity.

In this study, a low-cost code generation and recognition technology was introduced that uses a modified ejection system and deep learning-based object detection and classification technique. Products always have ejector pin (EP) marks in the die-casting process. If the head of the EP is modified to show directionality, and five or six EPs rotate dependently each time a product is produced, then the product has a unique EP pattern. This pattern can then be converted into a code using the deep learning-based object detection and classification technique.

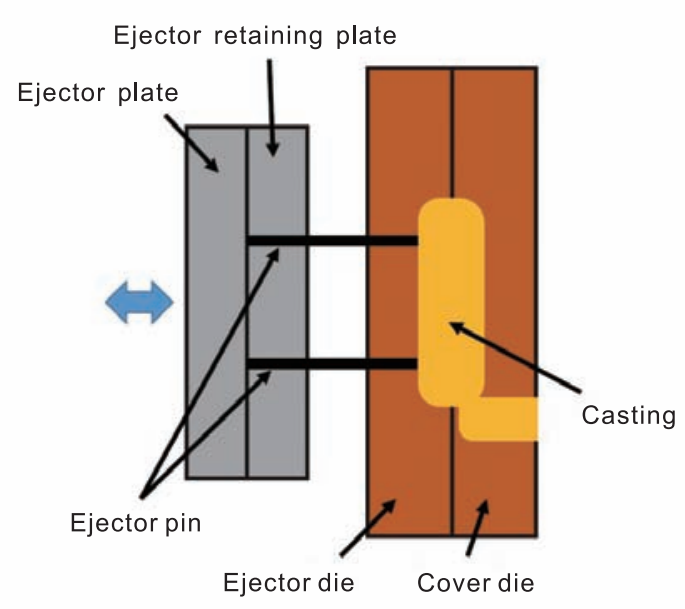

\section{Modified ejection system}

The ejection system serves to extract the solidified product in the final stage of the die-casting process ${ }^{[4]}$. The EP, which is an essential part of the ejection system, plays an important role in ejecting the product. The ejection system leaves EP marks on the product in the process of ejecting the solidified product. Typical ejection systems only have the ability to eject the product using EPs, as shown in Fig. 1(a). A new ejection system, Fig. 1(b), was developed that includes modified EPs and several functions to enable the generation of a code.

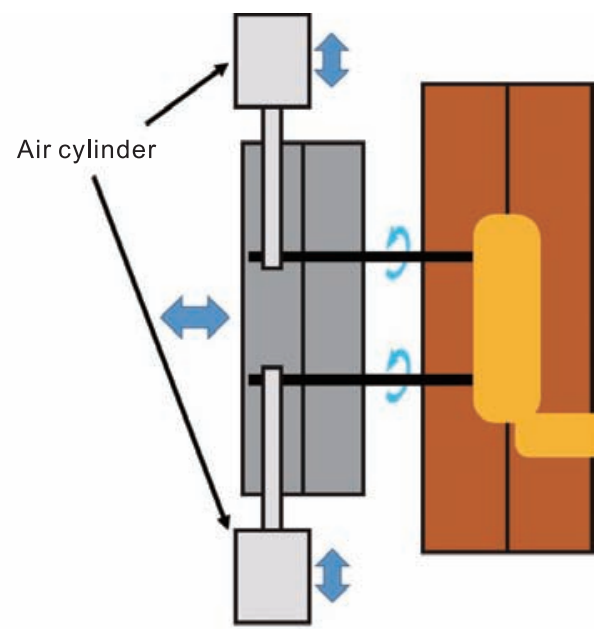

Fig. 1: Typical (a) and modified (b) ejection system

\subsection{Modified EP}

The first significant feature of our ejection system is the EP's shape. The EP's head is initially circular with no markings. The EP marks that are left on the die casting product are also circular (Fig. 2a). The style of the head of the EP was modified to one with a radius-length line such that the direction of the head can be seen. Therefore, when the ejection system ejects the product, the shape of the modified head remains on the product (Fig. 2b).

Generally, the EP diameter is proportional to the product size. EPs with 6 and $8 \mathrm{~mm}$ diameters were used for the products considered in this study. Even when EPs with less than $6 \mathrm{~mm}$ diameter (minimum $2 \mathrm{~mm}$ ) were used owing to the small size of the product, there was no problem in marking the product because the shape of the mark was simple.

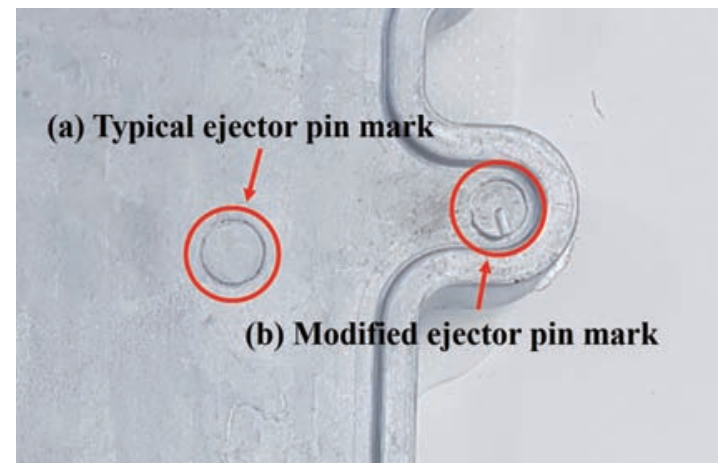

Fig. 2: Typical and modified ejector pin (EP) marks

\subsection{Rotation of EPs}

The second significant function is that the modified EP rotates dependently with a constant angle after ejecting the product. The modified ejection system includes air cylinders connected to EPs associated with code generation with a $6 \mathrm{~mm}$ latchet (Fig. 3). The cylinders are manipulated using a programmable logic controller (PLC) to allow the independently separated EPs to rotate dependently. The PLC was programmed to determine the order of rotation of the EPs and operate the cylinder to ensure its rotation with a constant angle according to the determined order. Therefore, the EP rotates dependently with a specific angle in accordance with a predetermined standard. Herein, the EP was implemented to rotate in $40^{\circ}$ increments.

Using the PLC, even if the die was replaced, only cylinders were connected to the PLC to ensure that the EPs can always rotate dependently in a similar manner.

Accordingly, when the ejection system ejects nine products, the first EP turns one turn and the second is rotated by $40^{\circ}$. Similarly, if the second EP goes around one turn, the third one is rotated by $40^{\circ}$. In this way, five or six EPs rotate dependently. Thus, each product has its own pattern of EP marks, which can be seen as a unique 5-6-digit code.

As the EPs rotate, one of the nine directions of the EP shape remains on the product. Of the nine shapes, 12 o'clock is zero, the shape rotated by $40^{\circ}$ clockwise from 12 o'clock is number one, the EP rotated by $80^{\circ}$ is number two, etc. Similarly, the numbers $0-8$ are expressed in $40^{\circ}$ increments. Therefore, 

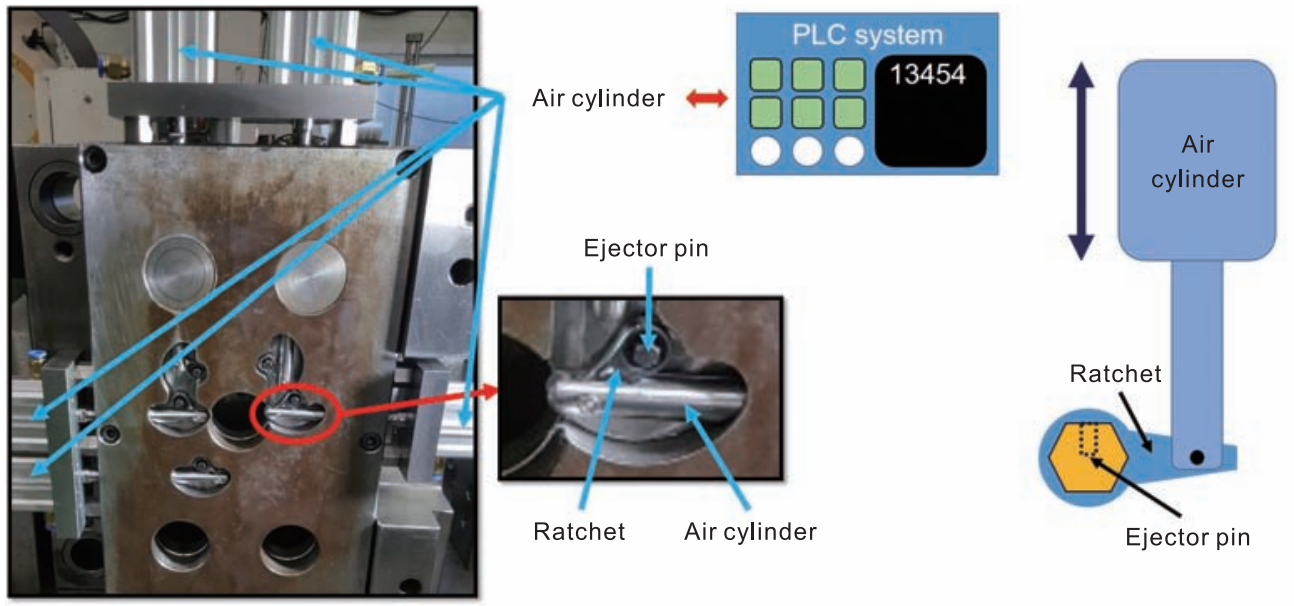

Fig. 3: Structure of modified ejection system

depending on the direction of the EP's mark, a nonary code is obtained (Fig. 4)

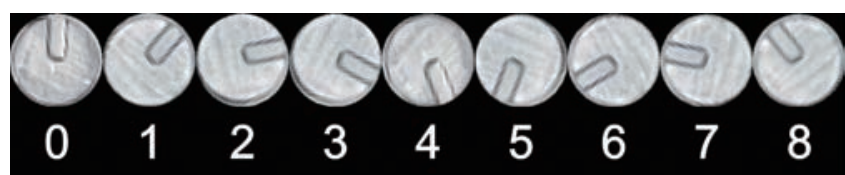

Fig. 4: Numbers along each direction

\section{Recognition program}

In Section 1, the generation of an EP code on a die-casting product using a modified ejection system was described. However, a problem of the ejection system was that there was an error in the initial angle of the EPs [called initial_error angle (IEA)] (Fig. 5). Because the product shown in Fig. 5 has a code 00001, all the EPs, except for the first digit in the red box, should point toward 12 o'clock and the EP in the red box should be rotated by $40^{\circ}$. However, a slight error can be noticed, except for the fourth digit in the green box. To solve this problem, a program that could calibrate the angle and convert it into a code was required. Therefore, a coderecognition program was developed to correct angles and convert them into a code using deep learning-based image detection and classification technology.

\subsection{EP detection model with YOLO-v3}

Object detection technologies ${ }^{[5]}$ include You Only Look Once (YOLO)-v3 ${ }^{[6-8]}$, region-based convolutional network $(\mathrm{R}-\mathrm{CNN})^{[9-11]}$, and single-shot multibox detector ${ }^{[12]}$. Herein, YOLO-v3 was adopted, which is faster and easier to operate than other models.

\subsubsection{Dataset}

Before training the EP detection model, the product image data must be labeled in the YOLO data format. Two types of products were photographed, 80 pcs each, and then labeled using the LabelImg ${ }^{[13]}$ program. The dataset was then organized by classifying the 160 labeled data into 112 training and 48 evaluation data.

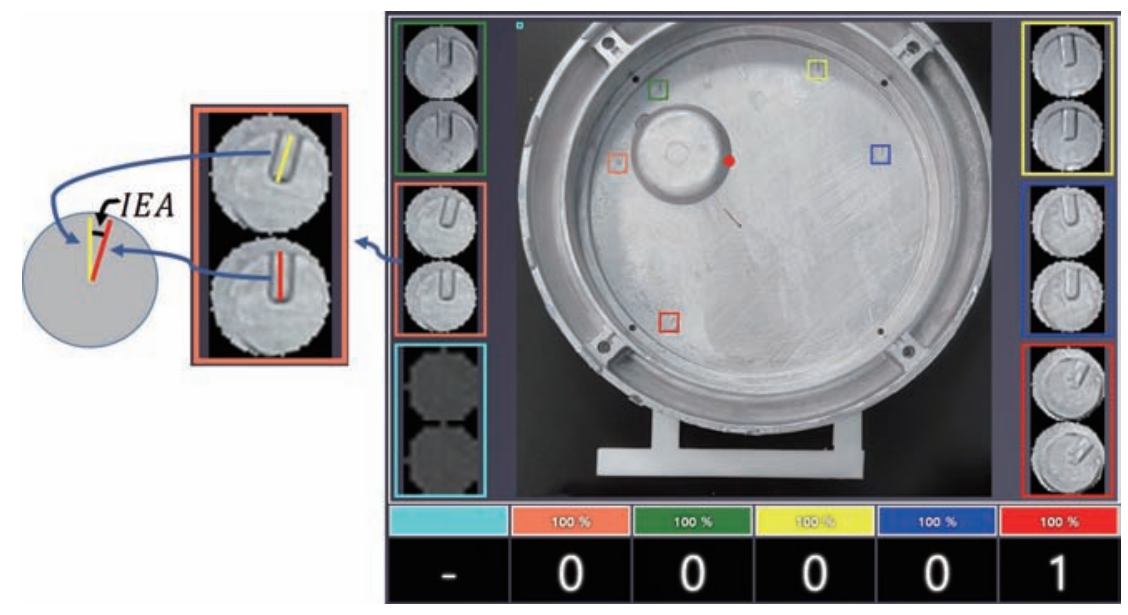

Fig. 5: Product in the image corresponds to the code 00001 . The EP in the red box should be rotated by $40^{\circ}$, and the rest should point toward 12 o'clock; however, slight errors exist, except for the EP in the green box. The top images in the colored boxes are EPs before the angle correction, and the bottom images show the corrected EPs 


\subsubsection{Training}

Recently, transfer learning ${ }^{[14-17]}$, which is a method to continue training a model pretrained from other existing data, has shown better performance. The YOLO-v3 model that was pretrained with the Common Objects in COntext (COCO) dataset ${ }^{[18,19]}$ was trained. The model that used transfer learning was compared with the model that had only learned our EP data. Results of the mean average precision (mAP) ${ }^{[20-22]}$ during 100 iterations of the model are shown in Fig. 6. Transfer learning showed considerably faster and more stable learning.
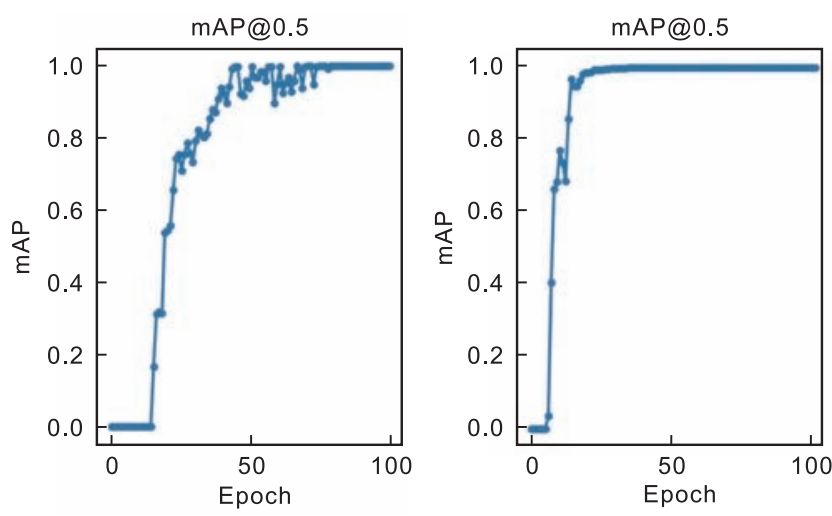

Fig. 6: The mAP results of the model that trained using only our EP data (a), and that used transfer learning (b)

YOLO gives information about bounding boxes that include EPs. It gives the center point and the dimensions of the bounding boxes and confidence scores as a probability value for whether the object being searched is inside the bounding box. Among the bounding boxes provided by YOLO, those with a confidence score $(0-1)$ higher than 0.5 were found without overlapping each other. The trained model detected the EPs very well with a high success probability (see Section 4 for detailed results).

\subsection{EP classification model with Resnet50}

\subsubsection{Dataset}

For the EP classification model, a dataset needed to be constructed by collecting data for each number. The EP

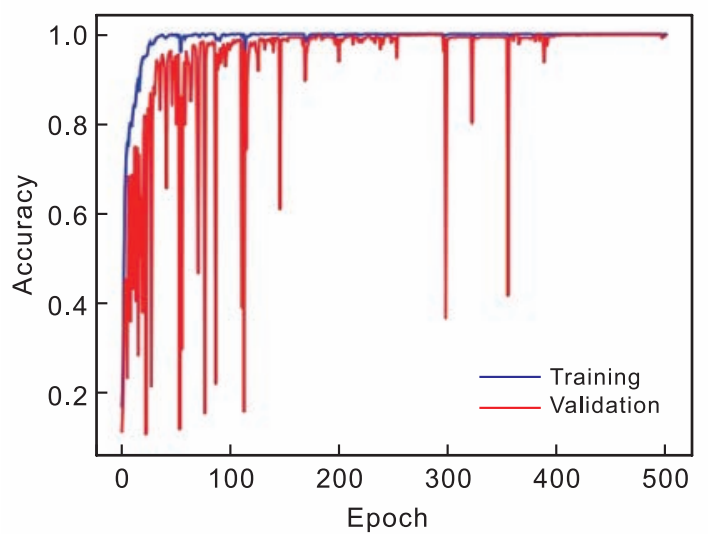

marks detected by the detection model trained previously were captured and utilized as data. Firstly, the number 0 was collected by rotating the captured images toward 12 o'clock. Then, they were rotated by $40^{\circ}$ to obtain $1,80^{\circ}$ to obtain 2 , etc. Thus, the nonary image data were collected. 1,260 training data, 140 per number, and 540 evaluation data, 60 per number, were organized. The feature size was $128 \times 128$.

\subsubsection{Training}

The residual network 50 (Resnet50) ${ }^{[23]}$, which is widely used in the image classification field, was employed. A model that used Resnet50 pretrained with the imagenet 1,000 dataset was compared with a model that had learned only our EP data. Figure 7 shows the training results. It can be seen that the transfer learning shows considerably better performance.

\subsection{Autorotation}

For autorotation, firstly, the EPs using the detection model learned earlier were located. YOLO gives the center points of the bounding boxes containing the objects. A barycenter (the red point in Fig. 8) of the points can be obtained. Next, the EP farthest from the barycenter and the angle $\theta$ between the segment $l$ connecting the EP to the barycenter and the $\mathrm{x}$-axis, with the barycenter as the origin, are obtained. This angle $\theta$ is called horizontal_correction_angle (HCA), and an HCA obtained by shooting in the correct direction is called initial horizontal_correction_angle (IHCA) (see the left image in Fig. 8). Finally, if the EPs were rotated by IEA-IHCA+HCA before classifying them using the classification model, the code could be recognized as if the products were always photographed from the correct direction. IEA and IHCA were obtained by an experiment using the first product (00001) that was photographed from the correct direction. The detailed method is described in Section 3.

\subsection{Automatic arrangement}

Another problem was that the detection model randomly detected the position of the EPs regardless of their sequence. Therefore, it was necessary to continually arrange them in a constant order. The barycenter obtained for autorotation was used for this purpose. Firstly, segments that connected the

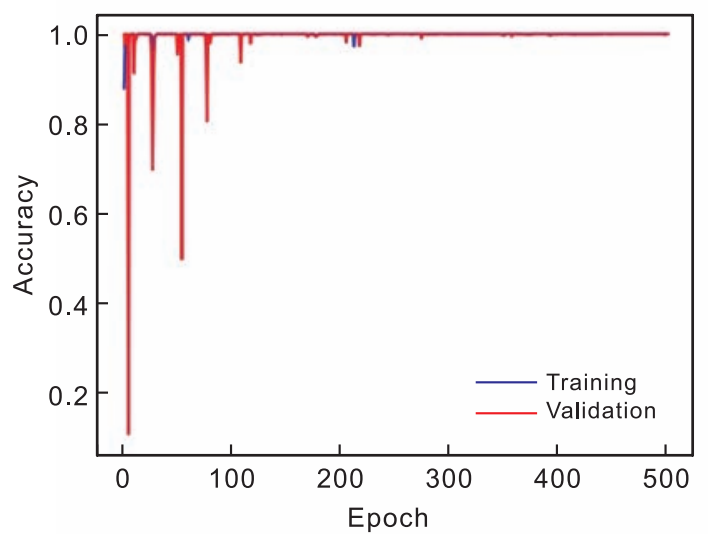

Fig. 7: Comparison of the models that trained using only our data (left) and that used transfer learning (right) up to 500 iterations 


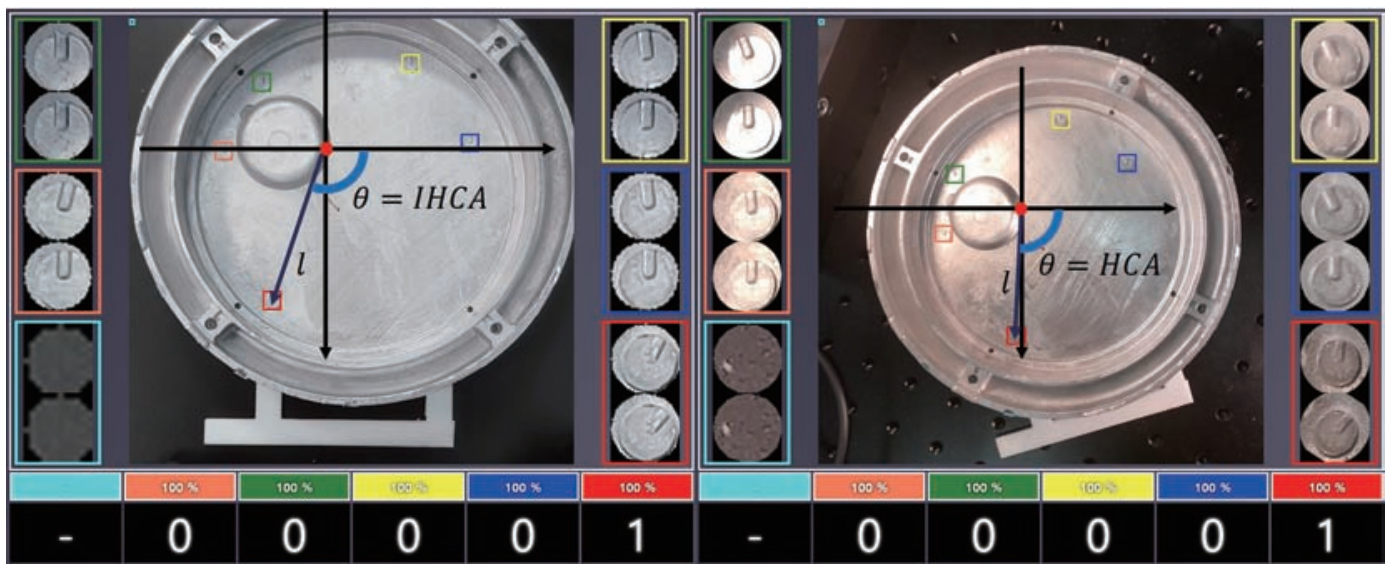

Fig. 8: EP with the longest distance from the barycenter (red point) is found and the angle $\theta$ between segment $I$ and the $x$-axis is obtained, with the barycenter as the origin. The angle $\theta$ obtained by shooting in the correct direction is IHCA (left). The top images in the colored boxes are the detected EPs, and the bottom images show the corrected EPs

barycenter to the EPs were found. Then the angles between the longest segment $l$ and the other segments were obtained. The EP corresponding to $l$ is the first digit (the red box in Fig. 9), and the remaining EPs were arranged counterclockwise in an order of segments with a small angle difference from $l$ (Fig. 9). Thus, EPs could always be arranged in a constant order.
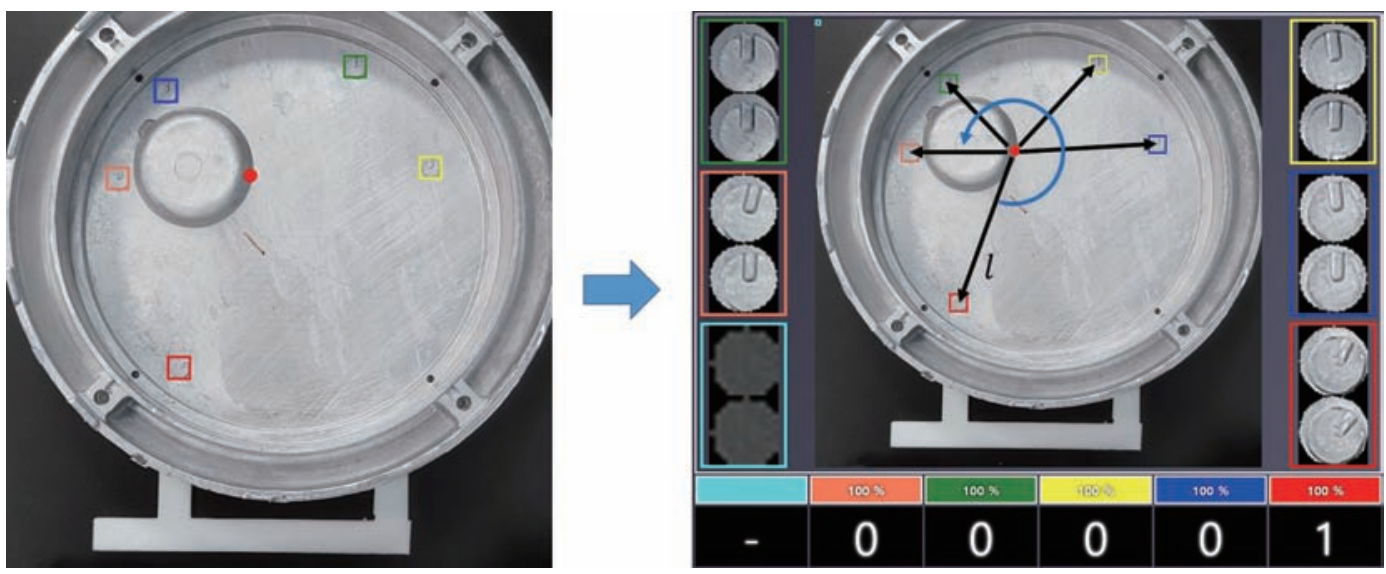

Fig. 9: YOLO finds EPs regardless of their sequence (left). They are arranged counterclockwise, starting with the EP (red box) that is furthest from the barycenter (right)

\section{Experimental}

The process of recognizing the EP code is summarized in Fig. 10.

Additional products not used for training but to test our modified ejection system and recognition program were produced. Twenty products of the first type, and thirty of the second type were manufactured. Firstly, it was verified whether the EP marks were well engraved on the casting product, and then a test was conducted to see if the EP marks were properly detected and converted into a code. All the 50 products were tested, but most of them demonstrated similar results; hence, this study only covers three products of the first type and four products of the second type among the additional products. The first products were produced to verify that the EPs were well marked, so the rotation angle did not follow a nonary sequence. Therefore, three products of the first type were used only for the marking and detection tests.
The second type of products were used for the marking, detection, and recognition tests. Before starting the test, firstly, the IEAs and IHCA were required. These values were obtained from the first product (00001) taken in the correct direction. Firstly, HCA was found for the first product. The HCA obtained is the IHCA for products of the second type. The EP in the red box was adjusted to be rotated by $40^{\circ}$ clockwise from 12 o'clock and the remaining EPs were controlled to point to 12 o'clock. The IEAs for each EP were obtained by comparing the difference with the detected EPs. The IHCA for the second type of product was $110^{\circ}$. The EP's IEA in the red box was $-15^{\circ}$, the IEA of the EPs in the blue and yellow boxes was $-8^{\circ}$, the EP's IEA in the green box was $0^{\circ}$, and the EP's IEA in the coral box was $12^{\circ}$. All future products of the second type would be autorotated by these values. Two recognition tests were conducted on each of the products. One 


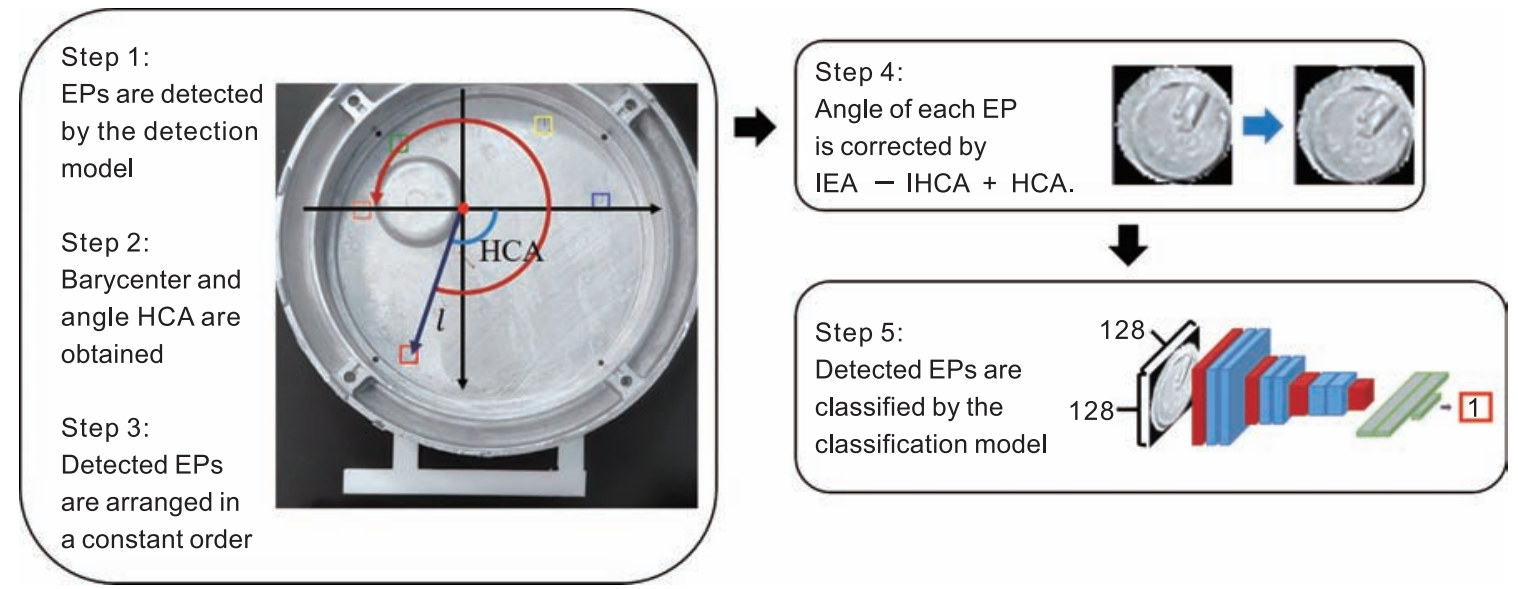

Fig. 10: EP code-recognition process

was photographed from the correct direction, and the other was photographed from the other direction.

\section{Results and discussion}

Three results were confirmed from the above experiment. The first is whether our modified ejection system correctly marks the modified EP mark on the product. As shown in Figs. 11 and 12 , our ejection system clearly marked the shape of the head of the modified EP on the die-casting product. The second is the result of the detection model. As shown in Figs. 11 and 12, the detection model detected the EPs successfully for all the seven products. Third, it was verified whether products taken in various directions can be successfully converted into code through recognition programs. In the code-recognition test using the second type, the EPs were correctly converted into code (Fig. 12). The recognition result was the same when it was taken from the correct direction and from a different direction (Fig. 12). YOLO is fast, but a known drawback is its lower accuracy for small objects. YOLO's third version, i.e., YOLO-v3, has considerably resolved this problem ${ }^{[7]}$. Regardless, there may be a decrease in detection accuracy because the EP was very small compared to the relative area of the product. Furthermore, small EP marks may be engraved when the size of the product decreases. However, the size of the EP marks does not change their shape; therefore, the proportion of the EP marks in the captured image is the only influential factor. In our experiments, when the size of the input of YOLO was $640 \times 640$, the EPs were detected successfully if the size of the EP was $16 \times 16$ or more. This result is expected to improve when training data of various sizes are used.

\section{Conclusion}

To improve the quality of the product in the die-casting process, it is necessary to understand the conditions under which a product is produced; the priority is to imprint a classification code on the product for individual recognition. Therefore, a modified ejection system that can generate a code and a program that can read the code have been introduced in this study. As shown in the above results, code generation and recognition using the modified ejection system demonstrated excellent recognition rates. Although problems still exist, such as the initial angle setting of the system, this concept can be used at a low cost in small and medium-sized companies. Following on from this, more diverse and complex products will be used for learning and testing. Image data from various products in more diverse environments are expected to further enhance the performance of our models. Moreover, reduction

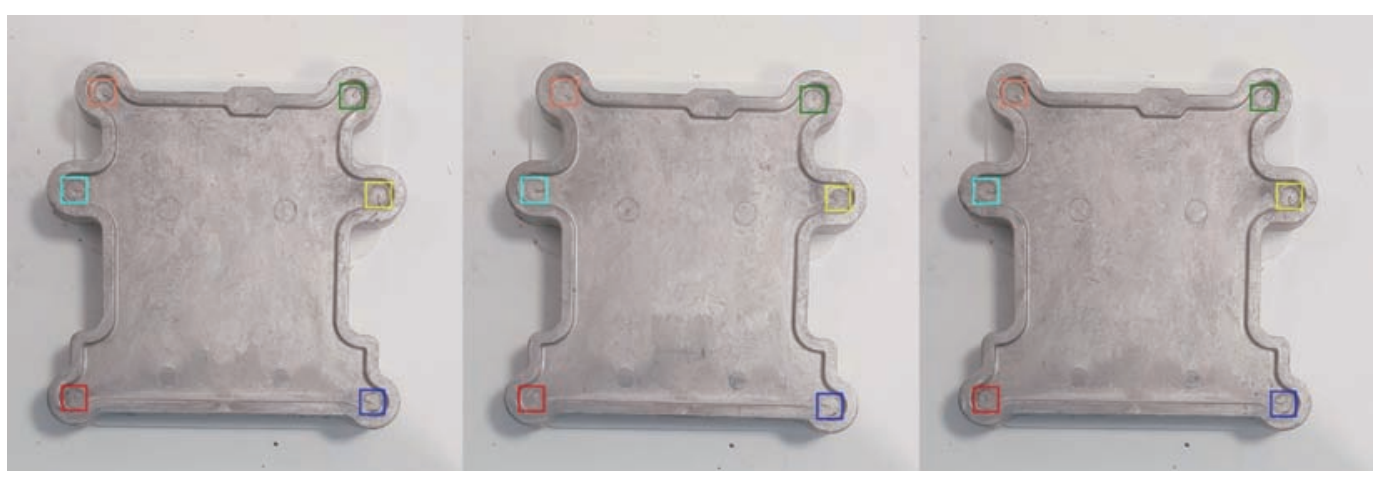

Fig. 11: Detection test for three products of the first type 

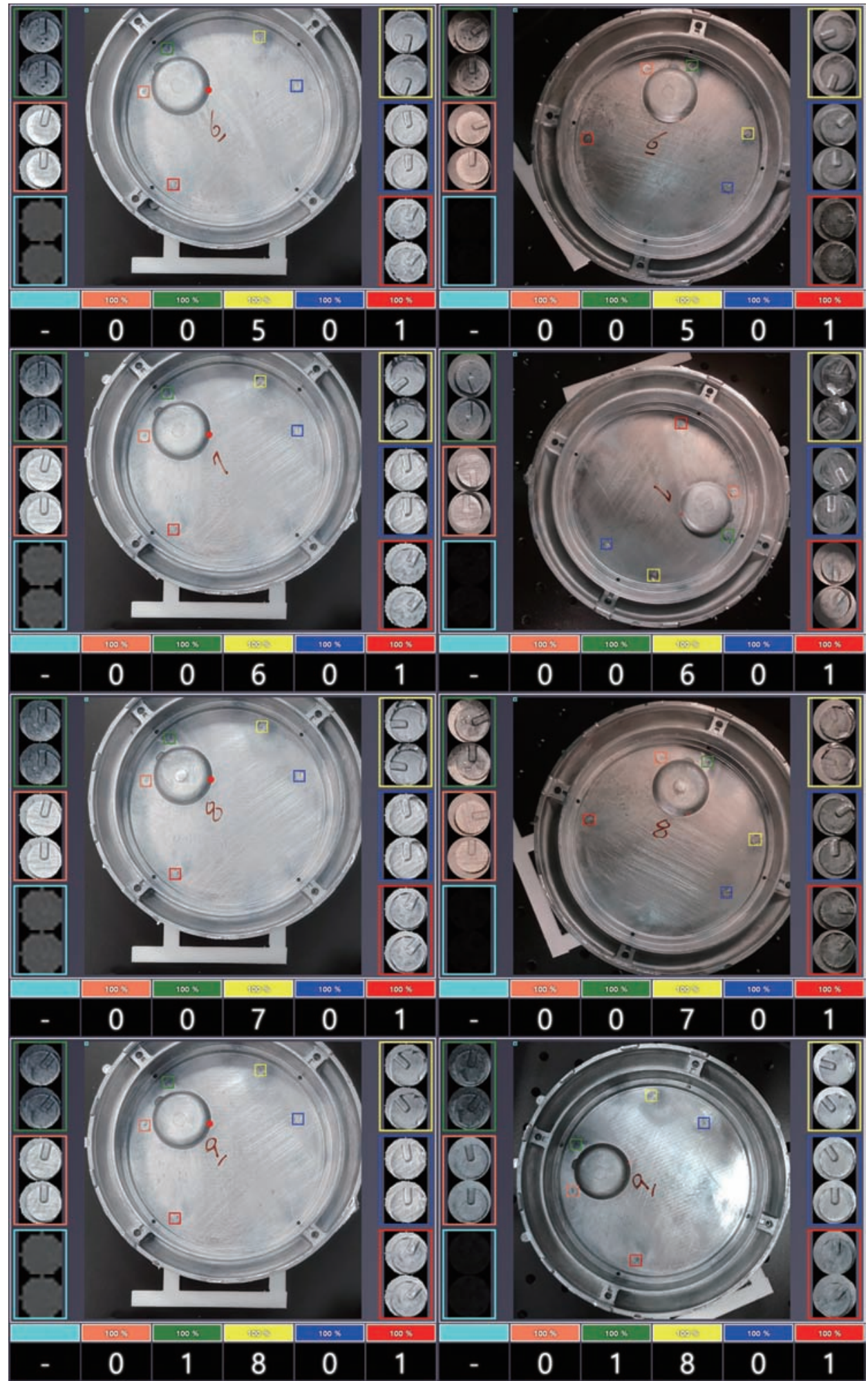

Fig. 12: Recognition test for four products of the second type. The images on the left are the results taken from the correct direction and those on the right are the results taken from different directions

of the initial angle error of the ejection system is expected to enable a faster and more straightforward recognition of the product than at present. In the future, research will be conducted to find the cause of the defect by managing the process data of the product using EP codes at a real manufacture site.

\section{Acknowledgments}

This research was supported by the development project of Industrial and Manufacturing Source Technology of the Korea Institute of Industrial Technology (KITECH), and granted financial resource by the Ministry of Economy and Finance, Republic of Korea (No. EO190031). 


\section{References}

[1] Fraser A, Maltais J, Hartlieb M, et al. Review of technologies for identification of die casting parts. In: Proc. NADCA Die Casting Congress, 2016.

[2] Fraser A, Brochu V, Gingras D, et al. Important considerations for laser marking an identifier on aluminum. Light Metals, 2016: 259-264.

[3] Fraser A and Hartlieb M. Traceability and laser marking of die castings. Die Casting Engineer, 2018: 16-21.

[4] Fanovo. Die Casting. http://fanovo.com/die-casting/Accessed February 4, 2020.

[5] Liu L, Ouyang W L, Wang X G, et al. Deep learning for generic object detection: A survey. International Journal of Computer Vision, 2019, doi: 10.1007/s11263-019-01247-4.

[6] Redmon J, Divvala S, Girshick R, et al. You only look once: unified, real-time object detection. Proceedings of the IEEE Computer Society Conference on Computer Vision and Pattern Recognition, 2016: 779-788.

[7] Redmon J and Farhadi A. YOLOv3: An incremental improvement, arXiv:1804.02767v1, 2018.

[8] Ultralytics L L C. YOLOv3 in PyTorch. https://github.com/ ultralytics/yolov3/Accessed February 4, 2020.

[9] Girshick R. Fast R-CNN. In: Proceedings of the IEEE International Conference on Computer Vision, 2015: 1440-1448.

[10] Girshick R, Donahue J, Darrell T, et al. Region-based convolutional networks for accurate object detection and segmentation. IEEE Transactions on Pattern Analysis and Machine Intelligence, 2016, 38(1): 142-158.

[11] Ren S, He K, Girshick R. et al. Faster R-CNN: Towards realtime object detection with region proposal networks. IEEE Transactions on Pattern Analysis and Machine Intelligence, 2017, 39(6): 1137-1149.

[12] Liu W, Anguelov D, Erhan D, et al. SSD: Single shot multibox detector. Lecture Notes in Computer Science (Including Subseries Lecture Notes in Artificial Intelligence and Lecture Notes in Bioinformatics), 2016, 9905 LNCS: 21-37.
[13] Tzutalin. Labellmg. https://github.com/tzutalin/labellmg/ Accessed February 4, 2020.

[14] Krishna S T and Kalluri H K. Deep learning and transfer learning approaches for image classification. International Journal of Recent Technology and Engineering, 2019, 7(5): 427-432.

[15] NVIDIA. What is transfer learning? https://blogs.nvidia.com/ blog/2019/02/07/what-is-transfer-learning/ Accessed February 4, 2020.

[16] Tan C Q, Sun F C, Kong T, et al. A survey on deep transfer learning. Lecture Notes in Computer Science (Including Subseries Lecture Notes in Artificial Intelligence and Lecture Notes in Bioinformatics), 2018, 11141 LNCS: 270-279.

[17] Weiss K, Khoshgoftaar T M, and Wang D D. A survey of transfer learning. Journal of Big Data, 2016, 13(1). doi: 10.1186/s40537016-0043-6.

[18] Lin T Y, Maire M, Belongie S, et al. Microsoft COCO: Common objects in context. Lecture Notes in Computer Science (Including Subseries Lecture Notes in Artificial Intelligence and Lecture Notes in Bioinformatics), 2014, 8693 LNCS (Part 5): 740-755.

[19] COCO. http://cocodataset.org/\#home/ Accessed February 4, 2020.

[20] Hui J. mAP (mean Average Precision) for object detection. 2018, https://medium.com/@jonathan_hui/map-mean-averageprecision-for-object-detection-45c121a31173/ Accessed 4 February 2020.

[21] McCann S. Average precision. 2020, https://sanchom. wordpress.com/tag/average-precision/ Accessed 4 February 2020.

[22] Padilla R. Metrics for object detection. https://github.com/ rafaelpadilla/Object-Detection-Metrics/ Accessed 4 February 2020.

[23] He K M, Zhang X Y, Ren S Q, et al. Deep residual learning for image recognition. Proceedings of the IEEE Computer Society Conference on Computer Vision and Pattern Recognition, 2016: 770-778. 